\title{
Interesting and unusual cases of chronic abdominal pain-intermittent gastric volvulus
}

\author{
Sivasankaran Vadivel Kumaran, Thangavelu Pugazhendhi, Mohammed Ali
}

Department of Medical Gastroenterology, Madras Medical College \& Government Rajiv Gandhi Hospital, Chennai, India Email: Dr_Valky@yahoo.com, Pugaka@rediffmail.com, Medgemmc@gmail.com

Received 25 August 2012; revised 27 September 2012; accepted 3 October 2012

\begin{abstract}
Objective: Intermittent gastric volvulus is a rare disease that requires high index of suspicion for diagnosis and treatment. The incidence and prevalence is unknown, may be due to under reporting or under diagnosis. Gastric volvulus may be transient producing few symptoms. The Borchardt's Triad may be present only during an acute presentation. Common symptoms may mislead to diagnose a nonsurgical disease if an evaluation is not done, keeping in mind a possibility of gastric volvulus, even if a UGI scopy is normal. Cases may be submerged in the community being undiagnosed. Case Series: CASE 1: 21 yrs old male with intermittent abdominal pain for $1(1 / 2)$ yrs with marfanoid habitus, MVP and a normal UGI scopy. BMS revealed an Organo-Axial Volvulus and ligament laxity per-operatively. CASE 2: 65 yrs old diabetic female with vomiting and abdominal pain for 3 months and left sided pneumonitis. UGI scopy showed twisted gastric folds immediately below OGJ and inability to visualise antrum. BMS revealed mixed volvulus with paraesophageal herniation of distal stomach. Per-operatively there was laxity of ligaments with omental content alone within the diaphragmatic rent. Posterior retrocolic sub-mucosal gastrojejunostomy(pexy) was done for all cases. Conclusion: Gastric volvulus should be thought of in a case of chronic intermittent abdominal pain with normal baseline evaluation. A Chest X-ray and BMS should be done, at the time of symptoms.
\end{abstract}

Keywords: Intermittent Gastric Volvulus; Chronic Abdominal Pain; Barium Meal Series; Whirl Sign

\section{INTRODUCTION}

Chronic intermittent gastric volvulus is a rare disease that requires high index of suspicion for diagnosis and treatment. The incidence and prevalence is unknown, may be due to under reporting or under diagnosis. Gastric volvulus may be transient producing few symptoms.
The risk factors that precipitate torsion or volvulus are the laxity of the ligaments of stomach, anatomical defects or mechanical displacement of stomach from its anatomical site. The Borchardt's Triad may be present only during an acute presentation. Many cases occur with paraesophageal hernia or diaphragmatic eventration. The signs and symptoms of intermittent gastric volvulus include intermittent abdominal pain and distention, especially in the upper abdomen, and vomiting with progression to nonproductive retching. Because of the rarity of this disease, common gastrointestinal complaints may mislead the physician to diagnose a nonsurgical gastrointestinal disease if a detailed history, physical examination and complete evaluation are not done, keeping in mind a possibility of gastric volvulus, even if a Upper gastrointestinal scopy is normal. Cases may be submerged in the community being undiagnosed. Traditionally, it is diagnosed by seeing intrathoracic viscera in the chest radiograph, followed by a barium contrast study or upper gastrointestinal endoscopy. The whirl sign, which is an important characteristic of gastrointestinal volvulus, is rarely seen on computed tomography in gastric volvulus. We report two cases of chronic intermittent gastric volvulus that presented with intermittent abdominal pain lasting for 3 hrs postprandial and ball rolling movements after food intake. This should remind all physicians to maintain a high index of suspicion for this disease when treating patients with chronic intermittent abdominal pain with normal baseline evaluation and to seek surgical intervention once diagnosed. The signs and symptoms of gastric volvulus depend on the rapidity of onset and degree of rotation and obstruction.

\section{CASE HISTORY}

CASE 1: A 21 yrs old male was admitted with complaints of abdominal pain on and off for the past 1(1/2) yrs. Abdominal pain was at the Right hypochondrium, intermittent pricking pain, non-radiating, increased with food intake, partly decreased after $3 \mathrm{hrs}$. He perceived ball rolling movements in abdomen after food intake on 
and off. He had loss of weight of about $15 \mathrm{kgs}$ in the last $1(1 / 2)$ yrs with no other significant history. General examination showed marfanoid habitus. Cardiovascular system examination showed a mid-systolic click and a short systolic murmur. Abdomen examination showed suspicious bruit in the right paraumblical region. Other system examinations were normal. A provisional diagnosis of chronic abdominal pain-Acid peptic disease/ Superior mesenteric artery syndrome/Celiac axis compression syndrome with Mitral valve prolapse-Mitral regurgitation was made. Mesenteric Doppler study revealed normal origin of Celiac, Superior mesenteric artery and Inferior mesenteric artery. Ultrasonogram was normal. Upper gastrointestinal endoscopy was normal. Echo showed thickened mobile posterior mitral leaflet, mild mitral valve prolapse-anterior mitral leaflet and normal left ventricular function. Patient still had abdominal pain following food intake. So a barium meal series was done to rule-out any dynamic obstruction or motility disorder. Barium meal series revealed an Organo-Axial Volvulus (Figures 1-3). So the patient was taken up surgery. Per-operatively patient had laxity of ligaments of stomach, so posterior retrocolic sub-serosal gastrojejunostomy(pexy) was done.

CASE 2: A-65-year old female was admitted with complaints of vomiting of 6 months duration and abdominal pain of 3 months duration. She had vomiting on and off for the past 3 years, now persistent for the past 6 months, half to 1 hour after food intake, containing non-bilious food material. She had epigastric dragging, non-radiating pain which occurred immediately after food intake and

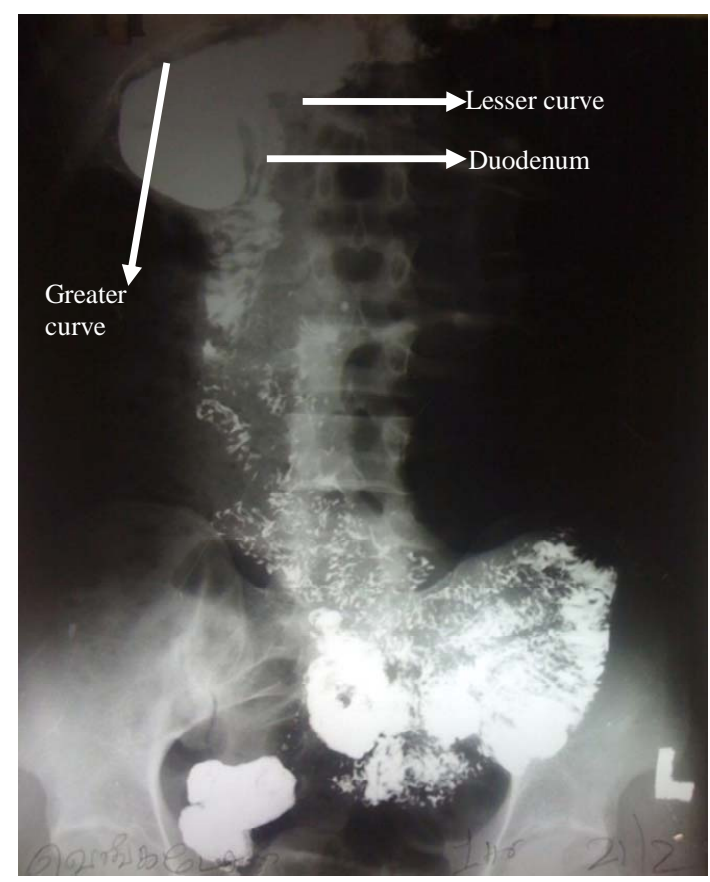

Figure 1. Organo axial volvulus.

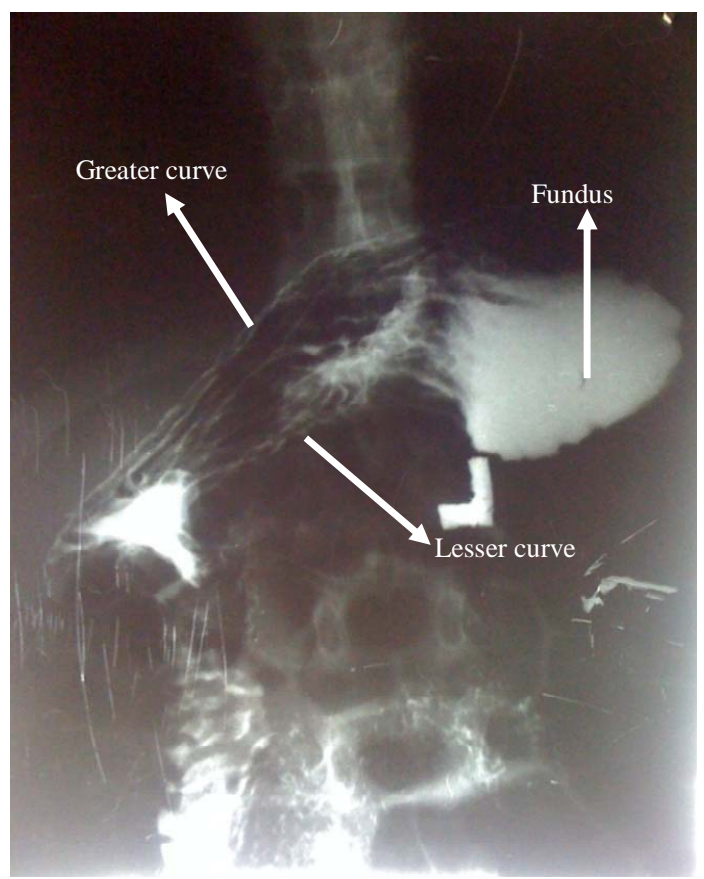

Figure 2. Organo axial volvulus.

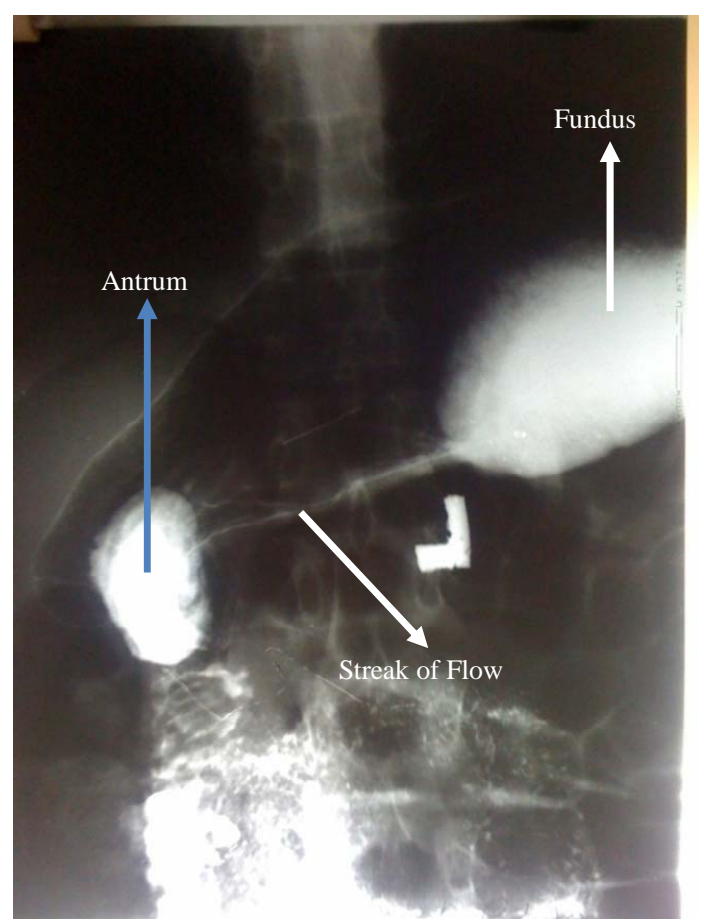

Figure 3. Organo axial volvulus.

relieved by vomiting. She had no ball rolling movements after food intake. She had loss of weight of nearly $6 \mathrm{kgs}$ in the past 1 year. She is a hypertensive for 4 yrs and diabetic for 3 yrs on drugs. General examination showed pallor. Respiratory system examination showed scattered crepts and wheeze on left side. Abdomen examination showed epigastric tenderness. Other system examina- 
tions were normal. A provisional diagnosis of peptic ulcer disease with or without gastric outlet obstruction/ diabetic gastroparesis with left sided pneumonitis was made. Haemoglobin was 7.8 gms\%. Chest X-ray revealed infiltrates in both hilum and left basal regions without any bowel shadows. Upper gastrointestinal endoscopy showed an inability to visualise the antrum in a routine manner with twisting of gastric folds (Figure 4) suggestive of gastric volvulus. Barium meal series revealed an organo-axial with mesentro-axial volvulus (Figures 5 and 6) with part of the stomach above the diaphragmatic shadow suggesting a paraesophageal herniation. So the patient was taken up for surgery. Peroperatively patient had laxity of ligaments of stomach with a small inverted " $\mathrm{v}$ " shaped rent in diaphragm of 3 cms with omental content which was reduced and closed, followed by a posterior retrocolic sub-serosal Gastrojejunostomy(pexy).

\section{DISCUSSION}

The term "volvulus" is derived from the Latin verb vol vere, which means to turn or roll. A brief History revolves around 3 B's - it was first described by Berti in 1866, First successful operation was done by Berg in 1896 and First delineation of classic triad of Borchardt in 1904. "Gastric volvulus" refers to the revolution of all or a portion of the stomach at least $180^{\circ}$ about an axis that causes an obstruction of the foregut. The obstruction may be acute, recurrent, intermittent, or chronic. A twisting or turning of the stomach of $180^{\circ}$ that results in only partial foregut obstruction is best defined as "gastric torsion" [1,2].

In adults, gastric volvulus is frequently associated with paraesophageal hernia (PEH) of the stomach. In our second case there was paraesophageal bowel herniation

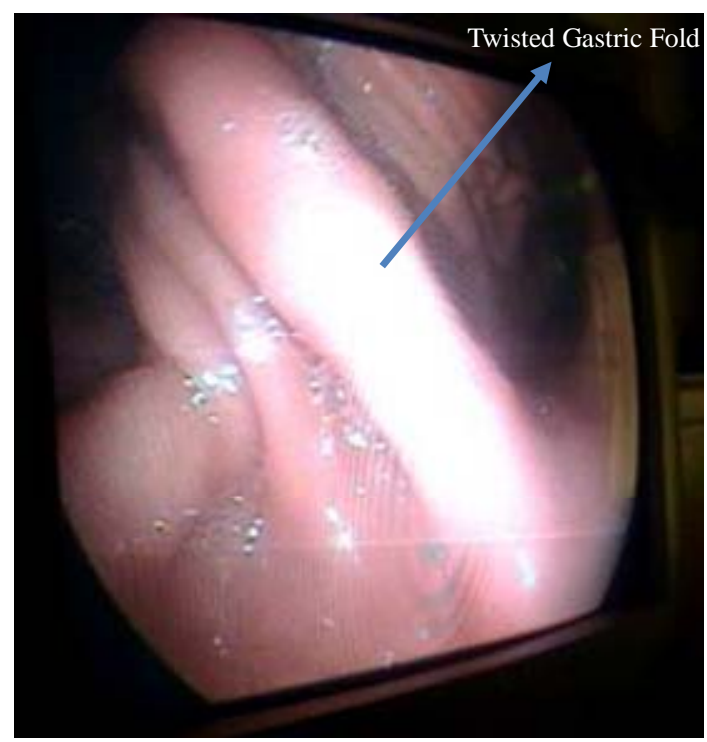

Figure 4. Twisted gastric folds on endoscopy.

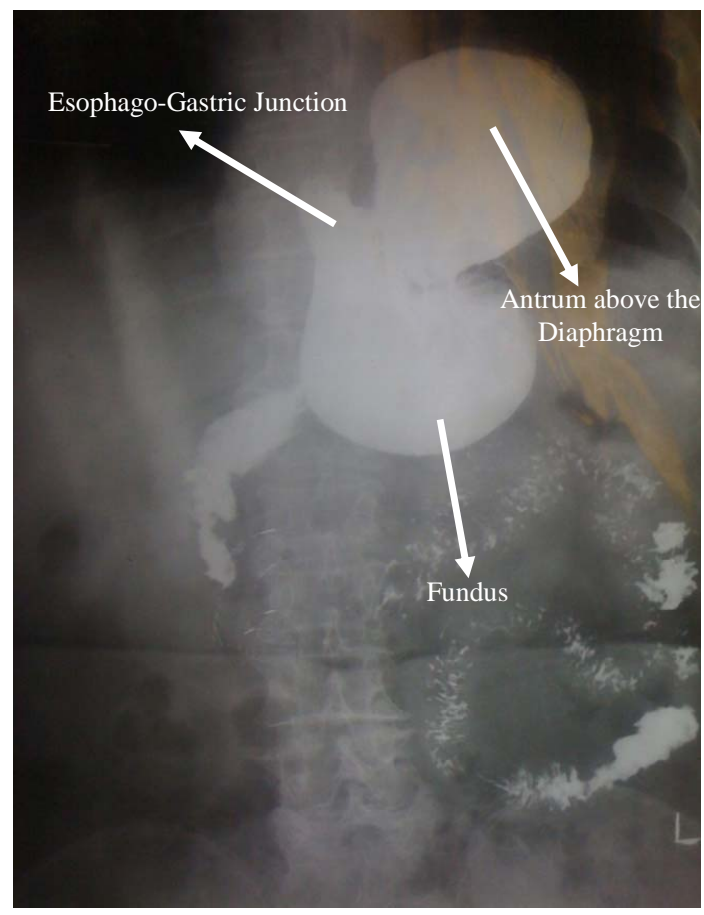

Figure 5. Mixed volvulus with part of loop above diaphragm.

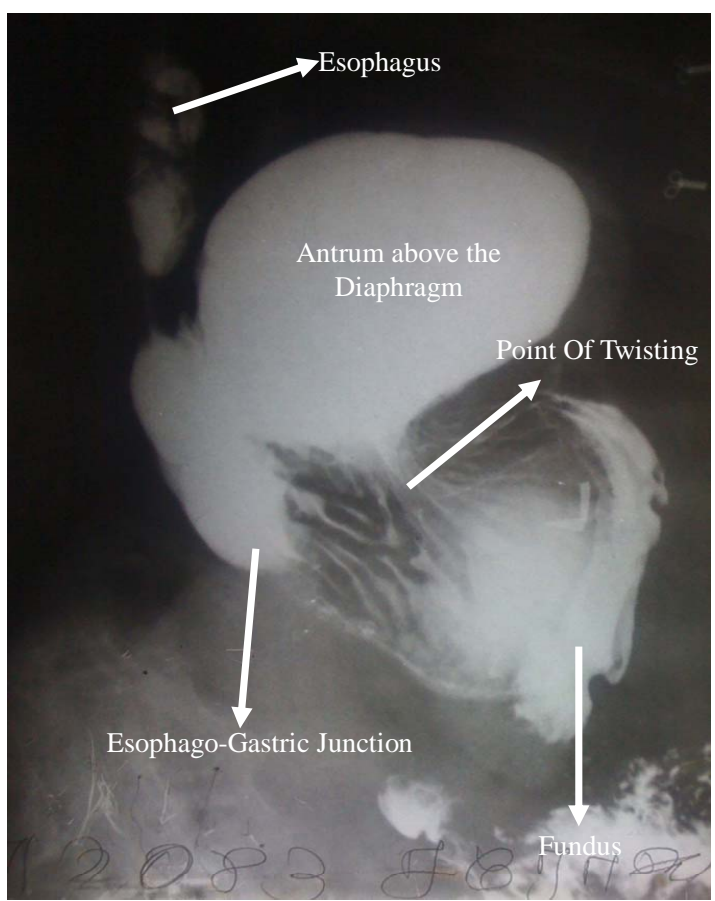

Figure 6. Mixed volvulus with part of loop above diaphragm.

on barium meal series which was the cause of left sided pneumonitis, which was confirmed per-operatively with the presence of omentum paraesophageally due to intermittent nature of gastric volvulus. The stomach is normally fixed to the abdominal cavity by 4 ligaments: the 


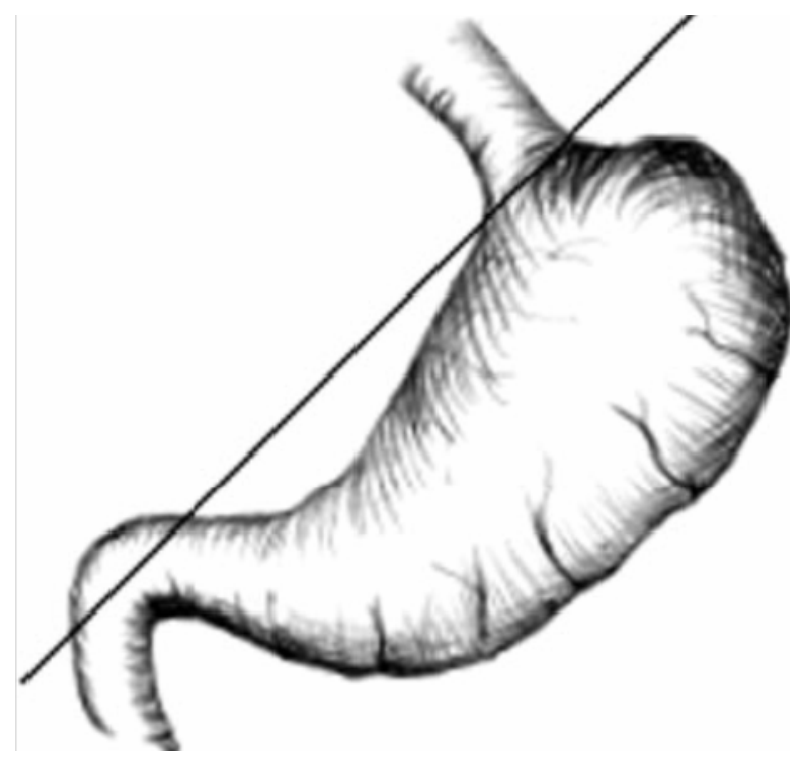

Figure 8. Longitudinal axis of rotation.

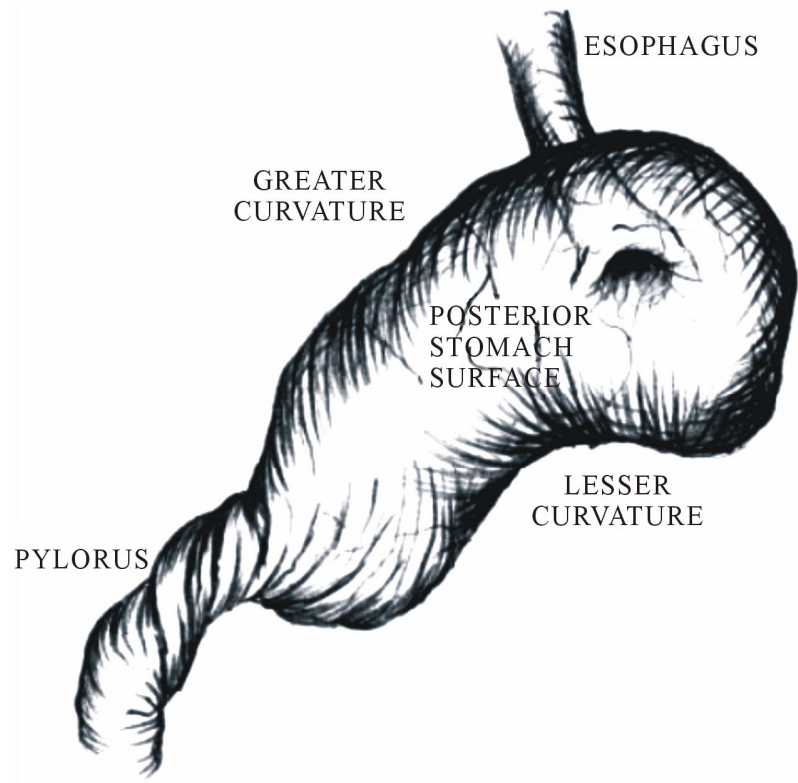

Figure 9. Organoaxial volvulus.

cases. The stomach in mesenteroaxial volvulus typically lies in a vertical plane, with the antrum and pylorus rotated anterior and superior to the gastroesophageal junction (Figure 11). Rotation of the stomach about both the organoaxial and mesenteroaxial axes is termed "combined volvulus" (Figure 12). Our second case had features of combined volvulus on barium meal series.

Gastric volvulus may be transient and produce few symptoms, or it may lead to obstruction and ischemia depending upon the severity and extent of rotation. Organoaxial volvulus is usually an acute event. Vascular compromise and gastric infarction may occur.

The incidence and prevalence of gastric volvulus are

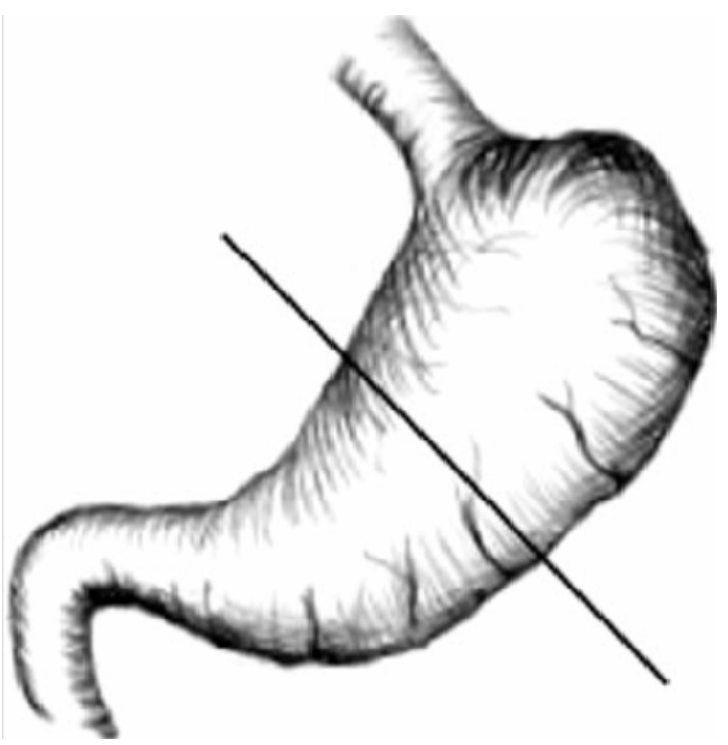

Figure 10. Mesentro axial axis of rotation.

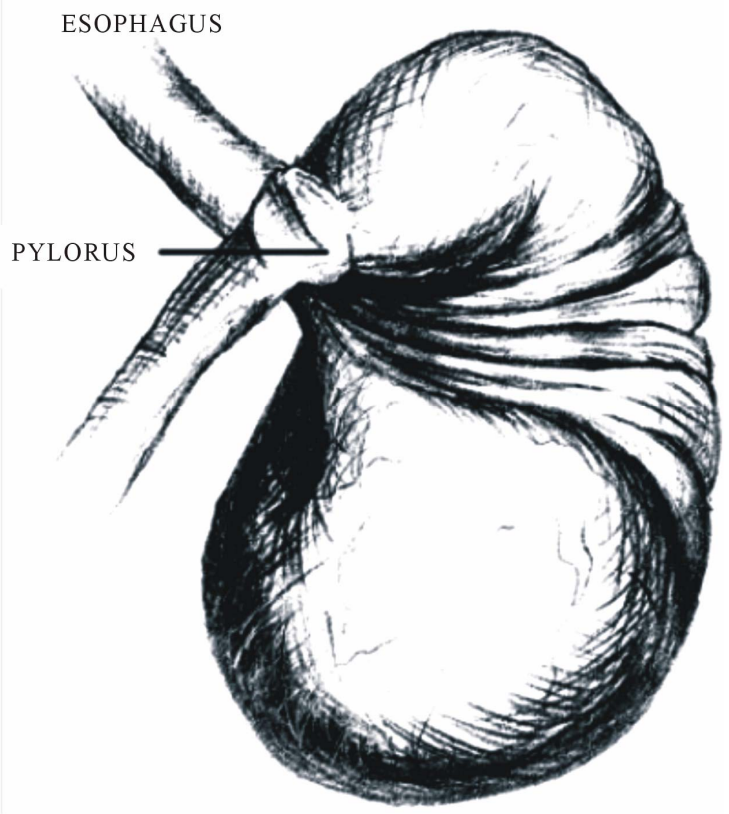

Figure 11. Mesentroaxial volvulus.

unknown with a peak incidence during the fifth decade of life [3]. It is difficult to estimate how many cases are intermittent and undiagnosed. Most often in association with a congenital diaphragmatic defect. Men and women are equally affected.

Less commonly reported is the chronic form of the disorder which has been seen with greater frequency in certain geographical areas, such as Japan and Saudi Arabia [4]. Our case report is on the less common form which may occur intermittently causing symptoms.

In cases of complete volvulus, it is impossible to pass a nasogastric tube into the stomach, abdominal pain, un- 


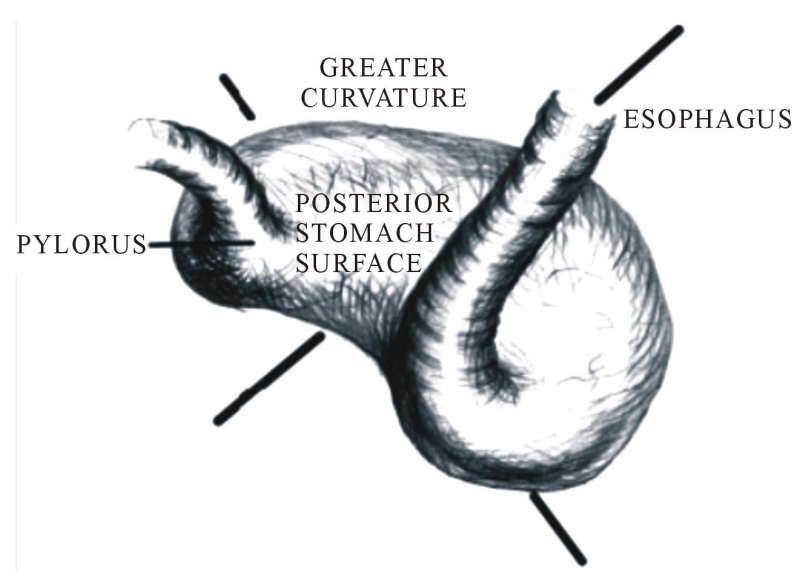

Figure 12. Combined volvulus.

productive retching-called Borchardt's triad. Upper endoscopy may show twisting of the gastric folds-in our second case it was evident at the time of endoscopy. A barium upper gastrointestinal radiograph will confirm the diagnosis-In our case it led us to diagnose the etiology of Chronic Abdominal Pain. Endoscopy is not prudent if gastric ischemia is suspected.

Chronic gastric volvulus is associated with mild and nonspecific symptoms such as dysphagia, epigastric discomfort or fullness, bloating, and heartburn, particularly after meals-in our case 1-patient presented with post prandial abdominal pain with ball rolling movements after food intake. A substantial number of cases are unrecognized as they occur intermittently. In our case 2patient presented with abdominal pain-which was relieved by vomiting.

\section{TREATMENT AND PROGNOSIS}

Acute gastric volvulus is an emergency. Nasogastric decompression should be performed if possible. If signs of gastric infarction are not present, acute endoscopic detorsion may be considered. Using fluoroscopy, the endoscope is advanced to form an alpha loop in the proximal stomach. Torque may then reduce the gastric volvulus. Surgery for gastric volvulus may be done by open or laparoscopic techniques. In recent years, there has been a trend toward laparoscopic repair. After the torsion is reduced, the stomach is fixed by gastropexy or tube gastrostomy. Gastric volvulus has also occurred after gastrostomy-tube placement by open, laparoscopic, and endoscopic techniques. Associated diaphragmatic hernia must be repaired. Combined endoscopic and laparoscopic repair or simple endoscopic gastropexy by placement of
Table 2. Management of gastric volvulus.

\begin{tabular}{ll}
\hline & $\begin{array}{l}\text { Endoscopic detorsion, if no sign of } \\
\text { gastric infarction with PEG }\end{array}$ \\
Acute Gastric & $\begin{array}{l}\text { Surgery-open or laparoscopy-gastropexy, } \\
\text { tube gastrostomy or subserosal } \\
\text { gastrojejunostomy. }\end{array}$ \\
\hline $\begin{array}{l}\text { Intermittent/Chronic } \\
\text { Gastric Volvulus }\end{array}$ & $\begin{array}{l}\text { Surgery-open or laparoscopy-gastropexy, } \\
\text { tube gastrostomy or subserosal } \\
\text { gastrojejunostomy. }\end{array}$ \\
\hline
\end{tabular}

a percutaneous gastrostomy (PEG) tube has been reported. Chronic gastric volvulus is treated in the same manner as acute volvulus (Table 2). Acute gastric volvulus has carried a high mortality in the past.

Gastrojejunostomy at the fixed point of the duodeno-jejunal junction tethers the untwisted stomach in the abdomen and prevents recurrent volvulus. This procedure carries the further advantage of preventing the not infrequent migration of the transverse colon into the chest anterior to the stomach. Fixation by anastomosis does not break down as may happen in simple gastropexy.

In our case we subjected the first patient for sub-serosal gastro-jejunostomy, to prevent further occurrence of torsion, which is a type of gastropexy performed for gastric volvulus.

We subjected the second patient for repair of an associated hiatus hernia after reducing the omental content and incomplete mixed volvulus with sub-serosal gastrojejunostomy, to prevent further occurrence of torsion.

\section{REFERENCES}

[1] Dalgaard, J.B. (1952) Volvulus of the stomach case report and survey. Acta Chirurgica Scandinavica, 103, 131153.

[2] Eek, S. and Hagelsteen, H. (1958) Torsion of the stomach as a cause of vomiting in infancy. Lancet, 1, 26-28. doi:10.1016/S0140-6736(58)92518-2

[3] Wastell, C. and Ellis, H. (1971) Volvulus of the stomach. A review with a report of 8 cases. British Journal of Surgery, 58, 557-562. doi:10.1002/bjs.1800580802

[4] Honna, T., Kamii, Y. and Tsuchida, Y. (1990) Idiopathic gastric volvulus in infancy and childhood. Journal of Pediatric Surgery, 25, 707-710. doi:10.1016/S0022-3468(05)80001-6 